\title{
The effect of minimally invasive sacroiliac joint fusion compared with sham operation: study protocol of a prospective double-blinded multicenter randomized controlled trial
}

\author{
Engelke Marie RANDERS ${ }^{1,2}$, Paul GERDHEM ${ }^{3}$, Jon DAHL ${ }^{1}$, Britt STUGE ${ }^{1}$, and Thomas Johan KIBSGÅRD ${ }^{1,2}$
}

\begin{abstract}
${ }^{1}$ Division of Orthopaedic Surgery, Oslo University Hospital, Oslo, Norway; ${ }^{2}$ Institute of Medicine, University of Oslo, Oslo, Norway; ${ }^{3}$ Reconstructive Orthopaedics, Karolinska University Hospital, Department of Clinical Sciences, Intervention and Technology, Karolinska Institutet, Stockholm, Sweden Correspondence: maeran@ous-hf.no

Submitted 2021-08-17. Accepted 2021-09-15
\end{abstract}

Background and purpose - The sacroiliac joint is increasingly recognized as a cause of pain in $15-30 \%$ of patients with low back pain. Nonoperative management is not always successful and surgical treatment with fusion of the joint is increasingly recommended. According to the literature, minimally invasive fusion reduces pain and improves function compared with nonoperative treatment. It is, however, unclear to what extent the placebo effect influences these results.

Patients and methods - The trial is designed as a prospective multi-center, double-blind, randomized shamsurgery controlled trial with 2 parallel groups. 60 patients with a suspected diagnosis of sacroiliac joint pain confirmed with sacroiliac joint injection are included according to the trial inclusion criteria. Patients are randomized with a 1:1 allocation into 2 groups of 30 patients each. The primary end-point is group difference in sacroiliac joint pain intensity on the operated side at 6 months postoperatively, measured by the Numeric Rating Scale. The main objective is to examine whether there is a difference in pain reduction between patients treated with a minimally invasive fusion of the sacroiliac joint compared with patients undergoing a sham operation.

Results - Unblinding occurs after the completed 6-month follow-up. The primary analysis will be performed when all patients have completed 6 months' follow-up. Follow-ups are continued to at least 2 years postoperatively. Data from the different groups will be compared based on the "intention to treat" principle.
Sacroiliac joint (SIJ) pain is increasingly recognized as a possible pain generator in low back pain (LBP) (1). In as many as $15-30 \%$ of patients with LBP, the SIJ may be the cause of pain, and to an even greater extent in patients suffering from "failed back surgery" $(1,2)$.

The SIJ transfers force from the spine through the pelvis to the lower extremities (3). Dysfunction of the joint's articular congruity, ligamentous structures, and motor control might be a cause of pain (4). The joint is richly innervated and contains mechano- and nociceptive receptors $(\mathbf{5 , 6})$. Even though pain can arise from the SIJ, the diagnosis of SIJ pain is challenging. Patient history and radiological imaging have low sensitivity and specificity for the diagnosis of SIJ pain $(7,8)$. Single clinical tests show little diagnostic strength for SIJ pain, but composites of clinical tests have fair sensitivity and specificity $(\mathbf{8}, 9)$. To strengthen the diagnosis, clinical tests are combined with intraarticular SIJ injection with local anesthetics (10).

SIJ pain can be severe, disabling, and reduce quality of life similarly to other spine conditions (11). Nonoperative treatment consists of physiotherapy, pain medication, intra-articular SIJ steroid injections, prolotherapy, and radiofrequency neurotomy of sacral nerve branches. There is limited evidence of effect of these treatment modalities (12-15). In many patients nonoperative treatment fails (16). Historically, open surgery has often been unsuccessful, due to intense postoperative pain and severe complications (17). Recent multiple minimally invasive (MIS) SIJ fusion studies have reported pain relief and improved function to a greater extent than nonoperative treatment and open surgery, and with low frequency of complications (16,18-20). The increase in both available implants for MIS SIJ fusion and their use is demonstrated by a growing number of publications. However, many are industry-sponsored and of poor quality (21-25). 
Although the literature suggests superiority of MIS SIJ fusion over nonoperative treatment, some of the effect may be explained by placebo; to what extent is not known. Therefore, we designed a non-industry sponsored prospective multicenter, double-blind, randomized sham-surgery controlled trial (RCT) in accordance with the SPIRIT recommendations to test the null hypothesis that there is no difference in pain reduction between sham surgery and MIS SIJ fusion.

\section{Patients and methods}

\section{Study design}

The trial is designed as a prospective multi-center, doubleblinded, randomized sham-surgery controlled trial with 2 parallel groups. Participants, investigators, and data analysts are blinded for group allocation until the primary endpoint. The primary end-point is group difference in SIJ pain intensity on the operated side at 6 months postoperatively, measured by the Numeric Rating Scale (NRS).

60 participants will be included after oral and written study information is given, and with written consent from the participant. There are 2 recruitment sites: Oslo University Hospital (OUS) in Norway and Karolinska University Hospital in Sweden. The recruitment phase started in April 2018, and the 1st participant was recruited in August 2018 in Norway and December 2019 in Sweden. Due to the coronavirus pandemic there have been large delays in inclusion in both countries. Inclusion is ongoing. At the time of writing (September 2021) 56 patients have been included. All patients are followed for 6 months before unblinding, and for a total of 2 years postoperatively. The study, including 6 months' follow-up of all patients, will probably be finished by April 2022.

\section{Patients}

Referrals from general practitioners and from departments of orthopedic surgery or physical medicine and rehabilitation are screened for eligibility by an orthopedic consultant in the 2 departments and all patients whose referral includes information concerning pain originating from the SIJ are included for evaluation. This baseline evaluation is performed at the hospital's outpatient clinics with standardized clinical examination in accordance with inclusion and exclusion criteria (Table 1). Those who fulfill the inclusion criteria are invited to participate in the RCT.

The orthopedic department at OUS is currently the only center in Norway that performs SIJ fusion. Approximately 40-60 patients are evaluated per year in the outpatient clinic. Sweden has evaluated twice the number of patients annually, and MIS SIJ fusion is performed at 2 centers.

\section{Interventions}

Patients are randomized to either receive MIS fusion of the SIJ or a sham procedure (Figure). All patients will receive the

\section{Table 1. Inclusion and exclusion criteria}

\section{Inclusion criteria}

1. Suspected SIJ pain for $>6$ months, or $>18$ months for pregnancy-induced pelvic girdle pain

2. Between 21 and 70 years old

3. Diagnosis of the SIJ as the suspected primary pain generator based on both of the following:

A. Pain pointed with a single finger (Fortin Finger Test) at or close to the posterior superior iliac spine (PSIS) with possible radiation into buttocks, posterior thigh, or groin

B. At least 3 of 6 clinical tests for SIJ pain (Laslett et al. 2005, Vleeming et al. 2008)

1. Compression

2. Posterior Pelvic Pain Provocation test-P4

3. Palpation of the long dorsal sacroiliac ligament

4. Patrick's FABER test

5. Active Straight Leg Raise (ASLR) test

6. Gaenslen's test

4. Reduced SIJ pain (NRS) of at least $50 \%$ of the pre-injection NRS score after fluoroscopically or computed tomography guided controlled injection of local anesthetic into the SIJ

5. Oswestry Disability Index (ODI) score of at least $30 \%$

6. SIJ pain of at least 5 on the Numeric Rating Scale (NRS), where 0 is no pain at all and 10 is worst imaginable pain

7. Bilateral SIJ pain, if one dominant side. If eligible the dominant painful SIJ will be treated in the study

8. Mentally and physically able to comply with study protocol

9. Signed study-specific informed consent

Exclusion criteria

1. Pain due to other causes, such as lumbar disc degeneration, lumbar disc herniation, lumbar spondylolisthesis, lumbar spinal stenosis, lumbar facet degeneration, and lumbar vertebral body fracture

2. Sacroiliac pathology caused by auto-immune disease (e.g., ankylosing spondylitis), neoplasia, or crystal arthropathy

3. History of recent $(<1$ year) fracture of the pelvis with documented malunion, non-union of sacrum or ilium, or any type of internal fixation of the pelvic rig.

4. Spine surgery during the past 12 months

5. Previously diagnosed or suspected osteoporosis (defined as T-score $<-2.5$ or history of osteoporotic fracture)

6. Documented osteomalacia or other metabolic bone disease

7. Any condition or anatomy that makes treatment with the iFuse Implant System infeasible

8. Patients with prior SIJ surgery

same pre- and postoperative assessment (i.e., blood samples, general anesthesia, draping, wound care, and pain medication). They will not be randomized before they are under general anesthesia. Patients are kept under general anesthesia for 40 to 50 minutes regardless of intervention group as this operation on average lasts for 45 minutes. The operations will be done by 1 or 2 surgeons and the theater will be closed to all other personnel. After the procedure is completed the participants will be treated with standard postoperative care as given after MIS SIJ fusion regardless of randomization group. The postoperative follow-up during the hospital stay will be done by health staff blinded to treatment allocation.

\section{Cases/surgery}

Patients randomized to the intervention group (cases) receive treatment with triangular titanium implants according to the 


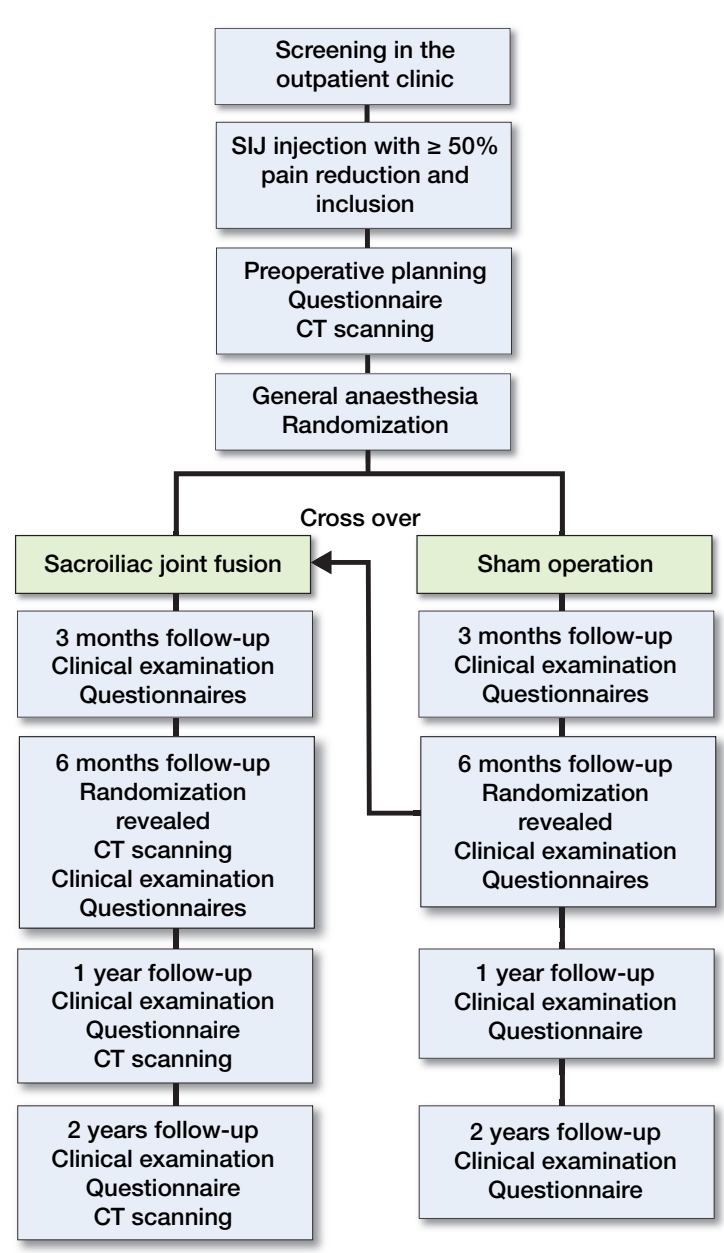

Flow-chart of trial timeline.

surgical technique manual (iFuse, SI BONE, Inc. CA, USA) (26). The procedure starts with a $3-5 \mathrm{~cm}$ long skin incision over the posterolateral aspect of the pelvis. Blunt dissection is performed through the subcutaneous tissue, fascia, and muscle. Guide pins are inserted over the SIJ at the desired entry-point, verified by fluoroscopy. The surgeon drills and broaches over the pins, and 3 triangular titanium implants are inserted. The wound is then closed. An injection of the SIJ with local anaesthetic is performed under guidance of fluoroscopy at the time of the procedure. A subcutaneous injection of local anaesthetic is given around the skin incision.

\section{Controls/sham surgery}

The control group consists of participants randomized to sham operation. The sham operation consists of the surgeon making the same skin incision as the surgical group receives. Blunt dissection is performed through the subcutaneous tissue, fascia, and muscle. Guide pins are inserted to the ileum, but do not enter the bone. The wound is then closed. Participants will be under general anesthesia while the procedure is simulated. All instruments needed are smeared with blood from the skin incision in order to keep the 2 procedures as similar as possible. An injection of the SIJ with local anaesthetic is performed under guidance of fluoroscopy during the procedure. A subcutaneous injection of local anaesthetic is given around the skin incision.

The surgeon will use a standard phrasing for the surgical report for both cases and controls. For controls, preoperative planned implants are reported and for intervention cases the actual implants used are reported.

Initially 2 methods of sham intervention (Norway vs. Sweden) were described in the protocol section on ClinicalTrials.gov. The reason for this was due to different criteria for obtaining ethical approval in the 2 countries. However, after observing the 2 different sham intervention methods the study group concluded that the differences were insignificant and therefore could be described similarly.

\section{Sample size}

The average mean preoperative NRS in previous studies has been reported to be $7.0-8.5(22,27-29)$. There are 2 RCTs that have compared MIS SIJ fusion with nonoperative treatment $(16,19)$. These report a mean decrease in global NRS of respectively 4.3 and $5.3(\mathbf{1 6 , 1 9 )}$. The same 2 studies showed a mean $0.5-1.3$ points decrease in NRS in the group treated with nonoperative treatment. We assume our trial's case group to have at least mean 3 points decrease in NRS and the control group to experience a mean 1 point decrease in NRS. 2 previous studies have suggested that 2 points' reduction in NRS represents a clinical important difference $(30,31)$. For the sample size calculation, we assumed a change that would result in a mean 2-point difference between the groups for the main outcome in NRS on the operated side 6 months postoperatively. The standard deviation (SD) was set to 2.5 points because the $\mathrm{SD}$ has been reported in previous studies to vary between 1.0 and 2.9 at 6 months' follow-up after SIJ fusion $(22,27-29)$. The probability of a type 1 error (alpha) was set to 0.05 . Based on these assumptions we calculate 25 participants in each group with $80 \%$ power using an online sample size calculator (www.clincalc.com). Due to a possible dropout of $20 \%$ we will include 30 participants in each group, giving 60 participants in the study.

\section{Ethics, registration, funding, and potential conflicts of interest}

Ethical approval for the study has been granted in Norway by the Ethics Committee Health Region Southeast, Oslo, Norway (2017/1892/REK sør- $\varnothing \mathbf{s t}$ A), and in Sweden from the Regional Ethics Committee in Stockholm, Sweden (nr: 2018/1463-31).

The protocol of this study is registered with clinicaltrials. gov (NCT03507049).

The final result from this study will be published in peerreviewed international journals and also disseminated through international congress lectures.

This trial has received funding from Sophies Minde AS in the form of salary payment for the fellow. The Division 


\section{Study procedures}

Randomization

Participants who give consent to participate in the trial and who fulfill the inclusion criteria will be randomized by the operating surgeon at each site ( 1 per site) after the participant has been anesthetized. Participants will be randomly assigned to either iFuse or sham operation. Randomization is performed as block randomization in blocks of 4 or 6 with a 1:1 allocation to fusion or sham surgery of the SIJ. Allocation is completed with a computer-generated randomization stratified by site through a specific website (Viedoc, Viedoc Technologies [35] provided by OUS). This website can only be accessed with personalized username and password, and only the operating surgeon has access to randomize participants.

\section{Blinding}

All other parties than the operating surgeon and assisting staff in the operating theatre are blinded as to which intervention the participant has received. This includes trial participants, care providers, investigators, and data analysts. No one other than the operating surgeons had access to randomization prior to follow-up. Unblinding occurs after completed 6-month follow-up. Participants who have undergone sham intervention and wish to cross over to surgical intervention are allowed to when the 6-month follow-up is completed. Both participants who were randomized to MIS SIJ fusion initially and participants who cross over to MIS SIJ fusion are then followed for at least 2 years postoperatively (Figure). Participants who have undergone surgical intervention with implants can undergo surgery on the opposite SIJ if they so wish after the initial 6-month follow-up.

Unblinding of participants may take place if there is a suspicion of a severe adverse event in which knowledge of implant positioning is essential for patient management. Examples of such events can be severe pain, new neurological deficits, or other injuries of such severity that they demand radiological examination to ensure adequate emergency medical care.

\section{Withdrawal}

The participant may withdraw from the study for any reason at any time. The investigators may also withdraw participants from the study in order to protect their safety and/or if they are unwilling or unable to comply with the required study procedures.

\section{Postoperative care}

All participants are advised to adopt partial weight-bearing on crutches for the first 4-8 weeks postoperatively. After 12 weeks there are no restrictions.

CT scan of the SIJ is performed preoperatively and at 6, 12, and 24 months' follow-up to evaluate proper implant placement and lack of radiological signs of loosening.

\section{Data analysis plan}

Data collection

Baseline data on age, sex, diagnosis of SIJ pain, level of SIJ pain, and response to injection if applicable is registered on all eligible patients evaluated at the outpatient clinic in order to evaluate reasons for failure to include patients and for exclusion.

All data from both trial centres is collected into the Viedoc website (35) provided by OUS. The data is confidential and not available as the trial is not complete and recruitment is still ongoing. Data collection is done either by direct electronic registration into the Viedoc database or on forms, subsequently transcribed into Viedoc by the investigators. If there is missing data this will be collected by contacting the participant or by medical records review. Any adjustment of data entry has to be documented and is registered and reported by the database.

\section{Data analysis}

Data from the intervention groups will be compared based on the intention to treat (ITT) principle (36). Statistical comparisons in order to test differences between 2 independent groups will be made by the Student's t-test or, in the case of a non-normal distribution, the Mann-Whitney U-test. In order to evaluate hypotheses of variables in contingency tables, the chi-square test will be used or, in the case of small expected frequencies, Fisher's exact test. In addition to this, descriptive statistics will be used to characterize the data. A p-value of < 0.05 will be considered as significant. A sensitivity analysis will be performed comparing the intention to treat data against the per-protocol data exclusively from patients who complied with the study protocol. All statistical analysis will be performed by a blinded statistician. The code for group belonging will not be broken until the analyses and interpretations of the results have been performed.

\section{Discussion}

MIS SIJ fusion is increasingly used worldwide with reported reduced pain, and improved physical function and quality of life compared with nonoperative treatment in two industrysponsored RCTs $(\mathbf{1 6}, \mathbf{1 9})$. Both showed a clinically and statistically significant difference between the surgically and nonoperatively treated groups with greater pain reduction in the surgically treated group $(\mathbf{1 6 , 1 9 )}$. It is a weakness that the studies were industry-sponsored. Further, a placebo effect might have contributed to the reported superiority of MIS SIJ.

To what extent a placebo effect influences the result after MIS SIJ fusion is not known. A placebo effect after surgery has been shown to be an important factor in efficacy (37). Both patient and health care provider can influence a placebo effect on treatment results. This has particularly been shown in treatment of chronic pain, where the psychological component is believed to be important. Patients with long-lasting 
diseases seem to be poorer placebo responders, but on the other hand placebos tend to work better in patients expecting to have changes in sensation of pain (38). Several sham controlled studies in orthopedic surgery have shown comparable pain relief in both groups, such as Moseley et al. (39) and Schrøder et al. (40). Hence, the effect of MIS SIJ fusion might be explained by placebo, but this is yet to be examined.

The ethical dilemma of sham surgery is relevant. To ask patients to undergo general anaesthesia for sham surgery might seem unethical. However, a German study of 1.37 million anaesthetic procedures with elective patients, graded as ASA classification physical status I and II, showed 7.3 cases of death or serious complication per million cases, corresponding to a very low risk (41). All types of surgery are associated with risks of complications, and so also for MIS SIJ (42). In the control group the risks for surgical complications should be very low. A possible result showing sham surgery to be equal to SIJ fusion might spare thousands of people from undergoing unnecessary elective surgery with the risk and costs such surgery entail. We therefore find it ethically acceptable to complete a sham-controlled double-blinded randomized prospective study.

TJK and JD conceived the study. All co-authors initiated the study design and contributed to the implementation of the study and to the refinement of the study protocol. ER drafted the manuscript. All authors have reviewed and given their final approval of the submitted version.

The authors gratefully acknowledge the assistance of the Division of Orthopaedic Surgery at Oslo University Hospital and Karolinska University Hospital for sponsoring and facilitating the trial.

1. Sembrano J N, Polly D W. How often is low back pain not coming from the back? Spine (Phila Pa 1976) 2009; 34: E27-E32.

2. DePalma M J, Ketchum J M, Saullo T R. Etiology of chronic low back pain in patients having undergone lumbar fusion. Pain Med 2011; 12 : 732-9.

3. Vleeming A, Schuenke M D, Masi A T, Carreiro J E, Danneels L, Willard $\mathbf{F} \mathbf{H}$. The sacroiliac joint: an overview of its anatomy, function and potential clinical implications. J Anat 2012; 221: 537-67.

4. Aldabe $\mathbf{D}$, Milosavljevic S, Bussey M D. Is pregnancy related pelvic girdle pain associated with altered kinematic, kinetic and motor control of the pelvis? A systematic review. Eur Spine J 2012; 21: 1777-87.

5. Sakamoto N, Yamashita T, Takebayashi T, Sekine M, Ishii S. An electrophysiologic study of mechanoreceptors in the sacroiliac joint and adjacent tissues. Spine (Phila Pa 1976) 2001; 26: E468-E71.

6. Szadek K M, Hoogland P V, Zuurmond W W, De Lange J J, Perez R S. Nociceptive nerve fibers in the sacroiliac joint in humans. Reg Anesth Pain Med 2008; 33: 36-43.

7. Elgafy H, Semaan H B, Ebraheim N A, Coombs R J. Computed tomography findings in patients with sacroiliac pain. Clin Orthop Relat Res 2001; (382): 112-18.

8. Vleeming A, Albert H B, Ostgaard H C, Sturesson B, Stuge B. European guidelines for the diagnosis and treatment of pelvic girdle pain. Eur Spine J 2008; 17: 794-819.
9. Laslett M, Aprill C N, McDonald B, Young S B. Diagnosis of sacroiliac joint pain: validity of individual provocation tests and composites of tests. Man Ther 2005; 10: 207-18.

10. Kennedy D J, Engel A, Kreiner D S, Nampiaparampil D, Duszynski B, MacVicar J. Fluoroscopically guided diagnostic and therapeutic intra-articular sacroiliac joint injections: a systematic review. Pain Med 2015; 16: 1500-18.

11. Cher D, Polly D, Berven S. Sacroiliac joint pain: burden of disease. Medical Devices (Auckland, NZ) 2014; 7: 73.

12. Luukkainen R K, Wennerstrand P V, Kautiainen H H, Sanila M T, Asikainen E L. Efficacy of periarticular corticosteroid treatment of the sacroiliac joint in non-spondylarthropathic patients with chronic low back pain in the region of the sacroiliac joint. Clin Exp Rheumatol 2002; 20: 52 .

13. Cohen S P, Hurley R W, Buckenmaier C C, Kurihara C, Morlando B, Dragovich A. Randomized placebo-controlled study evaluating lateral branch radiofrequency denervation for sacroiliac joint pain. Anesthesiology 2008; 109: 279-88.

14. Kim W M, Lee H G, Jeong C W, Kim C M, Yoon M H. A randomized controlled trial of intra-articular prolotherapy versus steroid injection for sacroiliac joint pain. J Altern Complement Med 2010; 16: 1285-90.

15. Patel N, Gross A, Brown L, Gekht G. A randomized, placebo-controlled study to assess the efficacy of lateral branch neurotomy for chronic sacroiliac joint pain. Pain Med 2012; 13: 383-98.

16. Polly D W, Cher D, Wine K, Whang P, Frank C, Lockstadt H, et al. Randomized controlled trial of minimally invasive sacroiliac joint fusion using triangular titanium implants vs. nonsurgical management for sacroiliac joint dysfunction. Global Spine J 2016a; 6: GP113.

17. Smith-Petersen M N, Rogers W A. End-result study of arthrodesis of the sacro-iliac joint for arthritisg: traumatic and non-traumatic. J Bone Joint Surge 1926; 8: 118-36.

18. Ledonio C G T, Polly Jr D W, Swiontkowski M F. Minimally invasive versus open sacroiliac joint fusion: are they similarly safe and effective? Clin Orthop Relat Res 2014; 472: 1831-38.

19. Sturesson B, Dengler J, Kools D, Pflugmacher R, Prestamburgo D. Sacroiliac minimal invasive fusion compared to physical therapy: sixmonth outcome from a multicentre randomised controlled trial. Spine J 2016; 16: S73-S74.

20. Whang P G, Darr E, Meyer S C, Kovalsky D, Frank C, Lockstadt $\mathrm{H}$, et al. Long-term prospective clinical and radiographic outcomes after minimally invasive lateral transiliac sacroiliac joint fusion using triangular titanium implants. Med Devices Evidence Res 2019; 12: 411-22.

21. Sachs D, Capobianco R. Minimally invasive sacroiliac joint fusion: one-year outcomes in 40 patients. Adv Orthop 2013; 2013: 536128.

22. Sachs D, Capobianco R. One year successful outcomes for novel sacroiliac joint arthrodesis system. Ann Surg Innov Res 2012; 6: 13.

23. Miller L E, Block J E. Minimally invasive arthrodesis for chronic sacroiliac joint dysfunction using the SImmetry SI Joint Fusion system. Med Devices (Auckl) 2014; 7: 125-30.

24. Rappoport L H, Luna I Y, G Joshua. Minimally invasive sacroiliac joint fusion using a novel hydroxyapatite-coated screw: preliminary 1-year clinical and radiographic results of a 2-year prospective study (clinical report). World Neurosurgery 2017; 101: 493.

25. Fuchs V, Ruhl B. Distraction arthrodesis of the sacroiliac joint: 2-year results of a descriptive prospective multi-center cohort study in 171 patients. Eur Spine J 2018; 27: 194-204.

26. SI BONE. iFuse surgical technique, 2020. Available from: https://si-bone. com/providers/solutions/ifuse/surgical-technique (Accessed Dec 3).

27. Kibsgard T J, Roise O, Sudmann E, Stuge B. Pelvic joint fusions in patients with chronic pelvic girdle pain: a 23-year follow-up. Eur Spine J 2013; 22: 871-77.

28. Duhon B S, Bitan F, Lockstadt H, Kovalsky D, Cher D, Hillen T, SIFI Study Group. Triangular titanium implants for minimally invasive sacroiliac joint fusion: 2-year follow-up from a prospective multicenter trial. Int J Spine Surg 2016; 10: 13. 
29. Polly D W, Swofford J, Whang P G, Frank C J, Glaser J A, Limoni R P, et al. Two-year outcomes from a randomized controlled trial of minimally invasive sacroiliac joint fusion vs. non-surgical management for sacroiliac joint dysfunction. Int J Spine Surg 2016b; 10: 28.

30. Farrar J T, Young J P, LaMoreaux L, Werth J L, Poole R M. Clinical importance of changes in chronic pain intensity measured on an 11-point numerical pain rating scale. Pain 2001; 94: 149-58.

31. Hägg $\mathbf{O}$, Fritzell $\mathbf{P}$, Nordwall $\mathbf{A}$. The clinical importance of changes in outcome scores after treatment for chronic low back pain. Eur Spine J 2003; 12: 12-20.

32. Copay A G, Cher D J. Is the Oswestry Disability Index a valid measure of response to sacroiliac joint treatment? Qual Life Res 2016; 25: 283-92.

33. Stuge B, Garratt A, Krogstad J H, Grotle M. The pelvic girdle questionnaire: a condition-specific instrument for assessing activity limitations and symptoms in people with pelvic girdle pain. Phys Ther 2011; 91: 1096-108.

34. EQ-5D Foundation, Euroqol Research. EG-5D-5L. About, 2017. Accessed Oct 5. Available from: https://euroqol.org/eq-5d-instruments/ eq-5d-51-about/.

35. Viedoc. 2021. Viedoc, https://www.viedoc.com (Accessed Sept 4).
36. Hollis S, F Campbell. What is meant by intention to treat analysis? Survey of published randomised controlled trials, BMJ 1999; 319: 670-4.

37. Turner J A, Deyo R A, Loeser J D, Von Korff M, Fordyce W E. The importance of placebo effects in pain treatment and research. JAMA 1994; 271: 1609-14.

38. Vandana R, Tushar R. Placebos: current status. Indian J Pharmacol 2001; 33: 396-409.

39. Moseley J B, Omalley K, Petersen N J, Menke T J, Brody B A, Kuykendall D H, Hollingsworth J C, et al. A controlled trial of arthroscopic surgery for osteoarthritis of the knee, N Engl J Med 2002; 347: 81-8.

40. Schrøder C P, Skare Ø, Reikerås O, Mowinckel P, Brox J I. Sham surgery versus labral repair or biceps tenodesis for type II SLAP lesions of the shoulder: a three-armed randomised clinical trial, Br J Sports Med 2017; 51(24): 1759-66.

41. Schiff J H, Welker A, Fohr B, Henn-Beilharz A, Bothner U, Van Aken $\mathbf{H}$, et al. Major incidents and complications in otherwise healthy patients undergoing elective procedures: results based on 1.37 million anaesthetic procedures. Br J Anaesth 2014; 113: 109-21.

42. Shamrock A G, Patel A, Alam M, Shamrock K H, Al Maaieh M. The safety profile of percutaneous minimally invasive sacroiliac joint fusion. Global Spine J 2019; 9: 874-80. 